\title{
28 Research Square \\ Effects of light qualities on growth, lipid contents and fatty acids profiles of Cylindrotheca closterium
}

Congying Zhang Zhang

Ningbo University https://orcid.org/0000-0002-5220-3980

Ruibing Peng ( $\nabla$ pengruibing@nbu.edu.cn )

Ningbo University

Yi Wu

Ningbo University

Zhihong Wu

Ningbo University

Zuhao Zhang

Ningbo University

Xiamin Jiang

Ningbo University

Zhen Xu

Ningbo University

Research

Keywords: light quality, growth, fatty acids, total lipids, Cylindrotheca Closterium

Posted Date: May 13th, 2021

DOl: https://doi.org/10.21203/rs.3.rs-486744/v1

License: (c) (1) This work is licensed under a Creative Commons Attribution 4.0 International License.

Read Full License 


\section{Abstract \\ Background}

Marine diatoms were considered as a promising alternative to microbial resources for the development of biofuels, food additives, medicines and chemical materials. Light of wavelength is a principal element that can easily be controlled in microalgal scale-culture and influence the growth, pigment composition, and lipids accumulation of algal cells. With the increasing implementation of light-emitting diodes (LEDs) in microalgal production systems (photobioreactors), a measure of light quality controlled by LED could be suitable to improve microalgal yields.

\section{Results}

In this study, Cylindrotheca Closterium, a widely used diatom, influenced by five light quality (white, red, blue, green and yellow light) was tested for its effects of growth rates, chlorophylls contents, total lipids contents and fatty acids compositions. The growth and chlorophyll experiments also showed that the green and yellow light significantly improve the growth rates and chlorophylls contents than other groups $(P<0.05)$. The group white light showed the greatest increases in total lipid contents of $C$. closterium $(P<$ 0.05). The group blue light had the highest polyunsaturated fatty acids (PUFAs) proportion $(P<0.05)$, while the group white showed the lowest PUFAs and the highest saturated fatty acids (SFAs) and monounsaturated fatty acids (MUFAs) proportions $(P<0.05)$. The proportions of SFAs and MUFAs were negatively correlated with growth, chlorophylls, and lipids. And the proportion of PUFAs and n-3 PUFA was positively correlated with growth chlorophylls, and lipids. Principal component analysis showed that the fatty acid composition differed among light quality groups.

\section{Conclusion}

In summary, green light and yellow light were conductive to boosting the growth and chlorophylls accumulation of $C$. closterium. White light increased total lipid yields, while blue light was superior in increasing the production of unsaturated fatty acids, especially on the timnodonic acid (EPA). The application of two-step methods to increase the production of biomass and fatty acids is an effective measure for the cultivation of $C$. closterium; green light is used to increase the growth, followed by white light cultivation to improve total lipids or blue light to enhance the proportion of PUFAs of $C$. closterium.

\section{Background}

With the rapid increase of the world population and the development of industrialization, energy sources in modern society have been constantly keeping increase in demand. However, the most extensively used and exploitable fossil energy is a non-renewable resource, which has been unable to meet the needs of economic and social development $[1,2]$. Therefore, the proposed search for renewable energy has 
become an important way to solve such problems. Compared with renewable resources such as wind, water or geothermal power, bioenergy is not only the oldest renewable energy but also the most novel solution, because of its advantages such as unlimited geographical conditions, easy access to sunlight, air and water resources. Moreover, biodiesel is one of the best promising candidates in response to the energy crisis, since it has the capability to minimize most of the environmental problems [3]. Microalgae are considered one of the most potential and healthy food due to their advantages such as small cultivation coverage areas, simple equipment requirements, fast growth rate, minimal consumption (they can be used as microalgal nutrients in municipal wastewater), and their high nutritive values[4, 5]. Microalgae species with superior performance have been currently developed, such as Chlorella and Nannochloropsis, while generally concentrated in green algae species [6, 7]. Diatoms, a few microalgae species that use fat as a direct reserve, have been invariably neglected. Research on diatoms has been aimed at the development of lipid extraction, which are rich in polyunsaturated fatty acids (PUFAs), making them the most optimal choice for high-added products. With the progress of microalgal sentiment and lipid extraction techniques, it is urgent for algal biologists to screen suitable microalgal strains, optimal environmental factors and methods to promote oil-yield improvements.

Light is the crucial source of energy input and feedback information for microalgae to maintain normal physiological activities, respond to external stimuli and adapt to their surrounding environment [8]. Generally, characteristics of light environments, including light quality, quantity and photoperiod, are extensively linked to the growth and development of diatoms, especially their photosynthesis [9]. Light quality (exclusive visible spectrum) is the wavelength range of a particular colour of light dispersed through a fixed medium. Wei et al (2020) also showed that light with wavelengths from $400 \mathrm{~nm}$ to 700 $\mathrm{nm}$ could be utilized for photosynthesis by microalgae. The responses of microalgal growth rates and lipid contents to differential light quality values were significantly diverse[8, 10]. Hence, microalgae require optimal light quality to achieve a maximum growth rate. Compared with light intensity and photoperiod, light quality is more easily controlled and regulated though generally limited, and has been abundantly applied in the cultivation of advanced plants[11, 12].

Cylindrotheca closterium is a marine eukaryotic unicellular diatom, and the cell shape is fusiform, $12 \sim 23$ $\mu \mathrm{m}$ in length and $23 \mu \mathrm{m}$ in width $[13,14]$. The microalgal species $C$. closterium is widely sought due to its ability to synthesize large amounts of lipid bodies and for its high biomass productivity, making it an ideal choice for the production of food, feed, nutraceuticals, and biofuel [15-17]. The investigation for the applicable ways to improve the yields and nutrient components of rhomboid algae, especially in PUFAs, has attracted many researchers. To provide additional information on light quality constraints influencing the production and lipid content, fatty acids composition of this diatom. Therefore, the objective of this study was to investigate the differential effects of $C$. closterium to different single LED wavelengths.

\section{Results}

\section{Comparison of the growth rates on various light qualities}


Different light qualities were used to stimulate $C$. closterium for 7 days aiming to observe their effects on growth. The treatment of light quality had significant differences on the growth rates of $C$. closterium $(P<$ 0.05; Fig. 1). Yellow Light (YL) (0.426) > Green Light $(\mathrm{GL})(0.425)>$ White Light $(\mathrm{WL})(0.398)>$ Blue Light $(B L)(0.396)>$ Red Light $(R L)(0.000)$. The dominance of $G L$ and $Y L$ was significantly greater than other three groups $(P<0.05)$.

\section{Comparison of chlorophylls content on various light qualities}

Figure 2 shows chlorophylls content of $C$. closterium under the different light quality; there were significant differences among treatments $(P<0.05)$. The range of chlorophyll a (Chla) content was from $1.26 \times 10^{-7} \mu \mathrm{g} \cdot$ ind $^{-1}$ to $1.99 \times 10^{-7} \mu \mathrm{g} \cdot \mathrm{ind}^{-1}$ under various light qualities. While chlorophyll c (Chlc) concentration was from $0.04 \times 10^{-7} \mu \mathrm{g} \cdot$ ind $^{-1}$ to $0.12 \times 10^{-7} \mu \mathrm{g} \cdot \mathrm{ind}^{-1}$ (Table. 2). Chla concentration on WL and GL was the highest, which was significantly higher than that under other light conditions $(P<$ 0.05). There was no significant difference in Chla content between $Y L$ and $B L(P<0.01)$, while the difference of various light qualities performing on Chlc content was consistent with Chla content.

\section{Comparison of total lipids content on various light qualities}

The determined total lipids content was demonstrated in Fig. 3. There were significant differences in lipids content between various light quality cultivation $(P<0.05)$. The total lipid contents ranged from $5.40-11.37 \%$, in which WL $(11.37 \%)>$ GL $(10.27 \%)>$ YL $(9.80 \%)>$ BL $(7.56 \%)>$ RL (5.40\%) (Table.2). The lipid content in WL condition was the highest, which was significantly higher compare with all the other light culture $(P<0.05)$. The total lipids content in groups $\mathrm{YL}$ and $\mathrm{GL}$ was significantly greater than that in groups $\mathrm{RL}$ and $\mathrm{BL}(P<0.05)$, but there was no significant difference in total lipids content between groups RL and $\mathrm{BL}(P>0.05)$.

\section{Comparison of fatty acids profile on C. closterium on various light qualities}

Light quality supplement had a significant effect on all measured fatty acids profiles and yield (Table. 3). Under light quality culture, the saturated fatty acid (SFA) concentration of $C$. closterium ranged from 26.77-33.65\%. No significant difference of GL, RL and BL on SFA's content was demonstrated in the presented experiment $(P<0.05)$. However, SFA under these treatments was significantly higher than that in groups WL and YL $(P<0.05)$. There was no significant difference in MUFA between LED RL and YL ( $P$ $<0.05$ ), both of which were significantly higher than those of other light quality treatments, ranging from 26.72-35.13\%. MUFA was mainly contributed by $\mathrm{C} 16: \mathrm{n}-7$, and the maximum content of MUFA can reach $37.50 \%$ under RL cultivation.

Polyunsaturated fatty acid (PUFA) content was not significantly altered between groups BL, YL and GL ( $P$ $<0.05$ ), while was significantly higher increase than that of WL and RL (from 28.86 to $39.95 \%$ ). It was mainly supplied by $C 20: 5(n-3)$ (EPA), as well as the highest part was $39.95 \%$ under blue light treatment. EPA and C22: 6(n-3) (DHA) were merely detected in n-3 PUFA. The content of n-3 PUFA in response to BL culture was significantly higher than other light quality treatments. No significant effect on the content of 
n-6 PUFA occurred with the administration of WL, GL and YL cultures, while was significantly higher than that in the RL and BL cultures. n-6 PUFA content ranged from $1.93-3.29 \%$ and was mainly composed of C20: 4(n-6) (ARA), C18: 2(n-6), and C16: 2(n-6). EPA's content under blue light culture (26.76\%), in C. closterium ranged from 18.21 to $26.76 \%$, was significantly higher compared with other light quality. All supplements with specific LED wavelength and optimal light intensity produced DHA yields of $C$. closterium, while DHA production remained a relatively low level, ranging from 1.93 to $3.29 \%$. Significantly increased DHA content was observed in RL culture compared with other light quality treatments, and there was no significant difference in the content of DHA with other experimental groups.

The principal component analysis (PCA) plots for the fatty acid composition of $C$. closterium, in which each point represented a sample stimulated by different light quality, showed quite evident differences among the different light quality treatments. The first and second principal components accounted for $64.6 \%$ of the variation ( $29.1 \%$ and $35.5 \%$ respectively) (Fig. 3). The fatty acid profiles were grouped into five clusters, which matched light quality groups. The fatty acid composition of group WL (W) and GL (G) was most closed, while the group RL (R) and BL (B) was most far away from each other. G's circle stretched across the four quadrants and had considerable proportion of C16: n-9. W circle had no significant enrichment in fatty acids composition. Furthermore, $\mathrm{C} 16: 3(\mathrm{n}-4)$ was the dominant composition of fatty acids in $\mathrm{YL}(\mathrm{Y})$ circle. $\mathrm{RL}$ and $\mathrm{BL}$ had their advantages and occupied respectively the fourth and third quadrant. $R$ circle was relatively rich in C18: 0 and C22: 6(n-3), while C14: 0, C20: 5(n-3) and C16: 4(n-1) were the dominant composition of fatty acids in B circle. These results indicated that the fatty acid composition of $C$. closterium under diverse light conditions could be differentiated.

\section{Correlation analysis of growth and fatty acids composition on C. Closterium}

The correlation coefficients $(r)$ of growth and fatty acids composition on $C$. Closterium ranged from -1 to 1 (Fig. 4). n-6 PUFA had no significant correlation with other measured indexes, so there was a bank space on the right side of Fig. $4(P<0.05)$. In the evaluation of quantitative index ' $r$ ', there was no correlation between SFA and Chlc, Chla; between lipids and MUFA, n-3 PUFA $(|r|<0.2)$. In addition, Chlc had weakly negative correlation with n-3 PUFA $(0.2 \leq|r|<0.4)$. Additionally, SFA were entirely moderately negative correlated with GR and lipids $(0.4 \leq|r|<0.6)$. Nevertheless, a moderately negative correlation was observed between MUFA and GR, Chlc; between PUFA and Chlc, lipids. Notwithstanding, n-3 PUFA had moderately positive correlation with GR and Chla $(0.4 \leq|r|<0.6)$. A strong negative correlation was found between MUFA and Chla $(0.6 \leq|r|<0.8)$. There was a very strongly positive correlation between PUFA and GR, Chla $(|r| \geq 0.8)$.

\section{Discussion}

Effects of different light qualities on the growth of C. closterium

The growth of $C$. closterium on groups $\mathrm{GL}$ and $\mathrm{YL}$ was greater than the other groups. The growth of $C$. closterium on groups $\mathrm{WL}$ and $\mathrm{BL}$ was moderate and the lowest growth was observed on the group RL. These results may be due to differences in spectral coverage, photon energy and luminous power among light qualities. Li reported that blue light could significantly promote the growth of Chaetoceros gracilis, 
while red light could cause growth inhibition [18]. Our results also showed that the main effect of the group $\mathrm{RL}$ was a decreasing trend in the growth rate of $C$. closterium.

Previous studies have suggested that mixed light such as white has more advantages light rather than the single wavelength for enhancing the production of microalgae $[19,20]$. WL has a wide range of wavelength containing various wavelength signals and energy, which can meet the requirements of the growth of microalgae. In the present study, group WL grew poorer than groups $G L$ and $Y L$, possibly due to the low proportion of certain important wavelengths. Compared with the single wavelength, there was a higher proportion of unsuitable wavelength in group WL than in the single wavelength groups and the more suitable wavelength may have been less absorbed by microalgae. Some studies demonstrated that the experiments on the effect of different ratios of red-blue mixed light on the growth of Spirulina had also achieved good results [20]. Other studies also showed that the experiment on the effect of different the ratio of red-blue mixed light on the growth of Spirulina has also achieved good results [20]. The effects of $C$. Closterium with different mixes of suitable wavelength is worthy of further research.

\section{Effects of various light qualities on chlorophylls content in C. closterium}

Photosynthesis occurs only in the chlorophyll-containing cells of green plants and algae, as it plays a key role in capturing photoelectrons and acts as an effective catalyst for photoreaction [21]. The observations of this experiment provided the evidence of significant effects of various light qualities on Chla and Chlc contents of $C$. closterium, and GL was the most suitable light source among these light qualities, which displayed the same trend on the growth of $C$. closterium. The key to the metabolism of microalgae lies in the conversion of light energy to chemical energy, and the photosynthetic pigment molecules carried by themselves can solve this problem very well [22]. Some studies have indicated that Chlorophyta and Cyanobacteria showed a preference for short wavelength, which may be related to their pigment composition $[23,24]$. The light energy absorption mode of Chlorophyta is mainly chlorophyll $a$ and $b$, which is more efficient for red light such as long wavelength, and the light system of cyanobacteria based on phycobilin can effectively absorb red light and blue light $[25,26]$.

Chla and Chlc, the main photosynthetic pigments of $C$. closterium, directly indicates the speed of the photosynthetic rate and is related to the growth of algal cells [27]. C.closterium to generally snuffcolouredcolored, rich in diadinoxanthin, lutein and other carotenoids, can greatly improve the efficiency of photoelectron capture and is important to assisting photocatalytic water splitting [28]. Consequently, there were favorable advantages of using the short wavelength light. The use of short-wave lengths, such as $\mathrm{GL}$ and $\mathrm{YL}$, prevailed for $C$. closterium. In addition, photosynthetic pigments have different light absorption with different wavelengths, which can affect the formation of photosynthetic pigments [29]. Kana indicated that environmental factors could affect the concentration and composition of photosynthetic pigments in microalgae in a dynamic manner [30]. Prasiola crispa, an aerial green-algae, has the long wavelength chlorophylls that can absorb considerable at approximately $710 \mathrm{~nm}$ [24].

Effects of various light qualities on lipid contents of $\mathrm{C}$. closterium 
Photons are one of the important factors affecting biochemical components in cultures, and various light quality conditions will have a significant impact on lipid synthesis [31, 32]. The results showed that in comparison to the other light qualities, the $\mathrm{WL}, \mathrm{GL}$ and $\mathrm{YL}$ cultivation were all conducive to the synthesis and accumulation of total lipids for $C$. closterium. Because of short-term stimulation of algal cultivation with light quality, RL stimulation did not damage algal cells; compared to growth experiments with longterm adaptation, those with RL stimulation could still result in growth. Hultberg found that Chlorella vulgaris with red and green light enhanced biomass production and fatty acid contents [33]. The methods of blue light use and nutrient starvation were also proved to be highly efficient in promoting the production of Chlorella sp. AE10 [10]. As mentioned above, certain studies have suggested that the total lipids of microalgal cells have distinct responses under different light quality treatments. Various light qualities have different effects on the activities of crucial enzymes in the lipid synthesis pathway, leading to total lipid content variation in microalgae $[9,34]$.

In this study, in comparison to the other light quality, group BL had no significant effects on lipid content and growth, which was not in agreement with the previous conclusions that BL would increase growth and lipid content $[23,25]$. These results seemed to suggest that $B L$, the shortest wavelength among the five kinds of light quality studied, had the highest energy with light irradiance and may cause high-energy damage to algal cells, which can be confirmed by the determination of antioxidant enzyme activity in algal cells in future experiments [35].

\section{Effects of various light qualities on fatty acid profiles of C. closterium}

Various light quality affected the fatty acid composition of $C$. closterium and influenced their growth and development. SFAs and MUFAs were negatively correlated with growth, while PUFAs were positively correlated with growth (Fig. 4). The PCA analysis of the fatty acid profile also showed several distinct differences under different light quality conditions (Fig. 5).

The main components of SFAs were myristic acid (C14: 0), palmitic acid(C16:0) and stearate acid (C18: 0 ) of $C$. closterium, and this was highest in group RL and lowest in group YL. Radakovits found that the accumulation of SFAs may be related to the expression level of acyl-ACP thioesterases in genetically engineered Phaeodactylum tricornutum [36]. Some studies also found that light quality can affect the activities of enzymes related to fatty acid synthesis, but whether red light can affect the activities of thioesterase and other related enzymes in SFA metabolic pathway [37]. We only confirmed that RL promoted the accumulation of much SFAs, which leads to a decrease in the proportion of PUFA, and so is the performance of group YL. However, which plays a major role in the synthesis of unsaturated fatty acids and saturated fatty acids needs to be further studied.

Some studies have shown that the synthesis of palmitoleic acid was correlated with substrate concentration of its palmitic acid (C16:0), but the current results did not support this view. Because group BL with the lowest MUFAs also had a high content of palmitic acid (21.33\%). However, there is a positive correlation between MUFA and growth, he relationship between growth performance and palmitoleic acid is still unclear. Other studies have pointed out that the existence of PUFA and MUFA is necessary for 
microalgae to maintain the stability of cellular membrane structure [38]. As the most important lipid reservoir of lipid droplets, the stability of the outer membrane structure of lipid droplets is related to whether it can effectively accumulate more fatty acids [39].

PUFAs with 18 and 20 carbon are vitally important substrates in the synthesis of long-chain unsaturated fatty acids [40]. The content of 16-carbon PUFAs (including C16: 2(n-6) and C16:3(n-4)) in was significantly higher the $Y L$ treatments than in all the other light treatments, and the high content of 16 carbon PUFAs suggested that YL may play a key role in inducing fatty acid biosynthesis-related regulation of the enzyme activities of prokaryotic plastids in algal cells. The WL treatment resulted in a greater increase in the 18 carbon (C18: 2(n-6) and C18: 3(n-6)) and 20 carbon (C20: 4(n-6), C20: 5(n-3) and C22: 6(n-3)) PUFAs contents; specifically, the content of ARA (2.89\%) and C18:2(n-6) (2.34\%) were significantly higher in the WL treatment than in the other treatments, which suggested that the composition of fatty acids in the WL treatment was consistent with the substrates of long-chain PUFA synthesis.

Although SFAs proportion limited the production of PUFAs under group BL, the EPA content still maintained the highest level compared that with in other treatments. EPA involved in the synthesis of phospholipids to form the cell membrane, can adjust the membrane fluidity to adapt to the stressful environment [41]. Accompanied by growing experiments, these observations may show that in group $B L$, C. closterium may be induced to produce light damage. Relative to selecting the optimal light intensity, BL possesses powerful emission energy and can activate the antioxidant system of algal cells. Nonetheless, in terms of light quality regulation, whether $\mathrm{BL}$ and $\mathrm{RL}$ were able to regulate the lipid metabolism process of diatom cells, the significant levels of $\mathrm{C16:2(n-6)}$ and $\mathrm{C} 16: 3(\mathrm{n}-4)$ in group $\mathrm{YL}$ showed that light quality also plays a regulatory role in the lipid metabolism process of diatoms. There was no significance or advantage in increasing the yield of fatty acids among the groups GL and WL. The process through which energy and information regulation played the main role should be determined by determining the key enzyme that regulates information and the chlorophyll light energy quenching experimental results of photosynthesis $[42,43]$.

\section{Conclusion}

Our study of $C$. closterium established that light composition played a dominant role affecting normal survival in this prominent marine species and economically aquatic microalgae and further enhanced our knowledge of the function of light factors. The application of two-step methods to increase the production of biomass and fatty acids is an effective measure for the cultivation of $C$. closterium. For example, green light is used to increase the growth, followed by white light cultivation to improve total lipids or blue light to enhance the proportion of unsaturated fatty acids of $C$. closterium.

\section{Materials And Methods}

\section{Microalgal strain and cultivation}


C. closterium was provided by the microalgae laboratory of Ningbo University. NUM3 (Table 1) was used as the culture medium using autoclaved seawater from Xiangshan Bay at $25 \%$ o salinity [44]. The culture medium was enriched with $1 \mathrm{~mL}$ of $1000 \times$ NUM3 nutrient solution. The culture of seawater and Erlenmeyer flasks was disinfected and sterilized. Stock cultures were grown in $1 \mathrm{~L}$ flasks and used to inoculate the working cultures (GXZ-intelligent light incubator, Ningbo Jiangnan Instrument Factory, China).

Table 1

Composition of NUM3 culture medium.

\begin{tabular}{|ll|}
\hline Nutrient composition & $\begin{array}{l}\text { Mass concentration } \\
\left(\mathbf{g} \cdot \mathrm{L}^{-1}\right)\end{array}$ \\
\hline $\mathrm{KNO}_{3}$ & 100 \\
$\mathrm{KH}_{2} \mathrm{PO}_{4}$ & 10.0 \\
\hline $\mathrm{FeSO}_{4} \cdot 7 \mathrm{H}_{2} \mathrm{O}$ & 2.50 \\
$\mathrm{MnSO}_{4}$ & 0.25 \\
$\mathrm{EDTANa}_{2}$ & 10.0 \\
$\mathrm{Na}_{2} \mathrm{SiO}_{3} \cdot 9 \mathrm{H}_{2} \mathrm{O}$ & 2.50 \\
\hline
\end{tabular}

Table 2

Characterization of cell density, RGR, Chla, Chlc and Total lipids properties of C. Closterium under different light quality culture.

\begin{tabular}{|c|c|c|c|c|c|}
\hline Treatment & $\begin{array}{l}\text { Cell density }\left(10^{4}\right. \\
\left.\text { ind } \cdot \mathrm{mL}^{-1}\right)\end{array}$ & $\begin{array}{l}\text { RGR } \\
\left(\text { day }^{-1}\right)\end{array}$ & $\begin{array}{l}\text { Chla } \\
\left(10^{-7} \mu \text { ind }^{-}\right. \\
\left.{ }^{1}\right)\end{array}$ & $\begin{array}{l}\text { Chlc } \\
\left(10^{-7} \mu \mathrm{g} \cdot \text { ind }^{-}\right. \\
\left.{ }^{1}\right)\end{array}$ & $\begin{array}{l}\text { Total lipids } \\
\text { (\% dry } \\
\text { basis) }\end{array}$ \\
\hline $\mathrm{RL}$ & $0.00 \pm 0.00^{c}$ & $\begin{array}{l}0.00 \pm \\
0.00^{c}\end{array}$ & $0.00 \pm 0.00^{d}$ & $0.00 \pm 0.00^{c}$ & $\begin{array}{l}5.40 \pm 1.15 \\
c\end{array}$ \\
\hline YL & $313.33 \pm 9.46^{a}$ & $\begin{array}{l}0.43 \pm \\
0.01^{\mathrm{a}}\end{array}$ & $1.26 \pm 0.17^{c}$ & $0.04 \pm 0.03^{b}$ & $\begin{array}{l}9.80 \pm 0.41 \\
\mathrm{ab}\end{array}$ \\
\hline GL & $313.33 \pm 25.04^{a}$ & $\begin{array}{l}0.43 \pm \\
0.01^{a}\end{array}$ & $1.99 \pm 0.05^{a}$ & $0.13 \pm 0.06^{a}$ & $\begin{array}{l}10.27 \pm \\
2.76^{a b}\end{array}$ \\
\hline$B L$ & $255.00 \pm 21.65^{b}$ & $\begin{array}{l}0.40 \pm \\
0.01 \mathrm{~b}\end{array}$ & $1.69 \pm 0.05^{b}$ & $0.09 \pm 0.04^{a b}$ & $\begin{array}{l}7.56 \pm 1.83 \\
\mathrm{bc}\end{array}$ \\
\hline WL & $255.00 \pm 15.21^{b}$ & $\begin{array}{l}0.40 \pm \\
0.01^{\mathrm{b}}\end{array}$ & $1.78 \pm 0.21^{\mathrm{ab}}$ & $0.12 \pm 0.07^{a b}$ & $\begin{array}{l}11.37 \pm \\
1.99 \mathrm{a}^{\mathrm{a}}\end{array}$ \\
\hline
\end{tabular}


Page 10/23 
Table 3

Proportions of fatty acids under different light quality cultivation of C. Closterium.

\begin{tabular}{|c|c|c|c|c|c|}
\hline \multirow{2}{*}{$\begin{array}{l}\text { Fatty Acid } \\
\text { Profile }\end{array}$} & \multicolumn{5}{|c|}{ Proportion (\%) } \\
\hline & RL & YL & GL & $\mathrm{BL}$ & WL \\
\hline C14: 0 & $5.41 \pm 0.32^{b}$ & $5.18 \pm 0.18^{b}$ & $6.99 \pm 0.35^{a}$ & $7.07 \pm 0.79$ a & $6.39 \pm 0.08^{a}$ \\
\hline C16: 0 & $\begin{array}{l}22.01 \pm 0.85 \\
\text { a }\end{array}$ & $17.22 \pm 0.85^{c}$ & $20.5 \pm 0.75^{a b}$ & $\begin{array}{l}21.33 \pm 0.59 \\
a\end{array}$ & $\begin{array}{l}19.56 \pm 0.99 \\
b\end{array}$ \\
\hline C18: 0 & $6.23 \pm 0.80^{a}$ & $4.38 \pm 0.15^{b c}$ & $5.05 \pm 0.37^{b}$ & $4.94 \pm 0.10 b c$ & $4.23 \pm 0.17^{c}$ \\
\hline SFA & $\begin{array}{l}33.65 \pm 1.47 \\
\text { a }\end{array}$ & $26.78 \pm 0.93^{c}$ & $32.55 \pm 0.74^{a}$ & $\begin{array}{l}33.35 \pm 1.36 \\
\text { a }\end{array}$ & $\begin{array}{l}30.19 \pm 1.09 \\
b\end{array}$ \\
\hline C16: n-7 & $\begin{array}{l}31.95 \pm 1.70 \\
a\end{array}$ & $29.72 \pm 1.74^{b}$ & $25.22 \pm 0.71^{c}$ & $\begin{array}{l}21.85 \pm 1.01 \\
d\end{array}$ & $28.14 \pm 0.79$ \\
\hline C16: n-9 & - & - & $0.93 \pm 0.02^{a}$ & - & - \\
\hline C18: n-7 & $4.88 \pm 0.24^{a}$ & $4.37 \pm 0.19^{a}$ & $3.66 \pm 0.41^{b}$ & $4.87 \pm 0.40^{a}$ & $4.84 \pm 0.32^{a}$ \\
\hline C18: n-12 & $0.67 \pm 0.06^{c}$ & $1.04 \pm 0.09^{b}$ & - & - & $1.68 \pm 0.19^{a}$ \\
\hline MUFA & $37.5 \pm 1.59^{a}$ & $35.13 \pm 1.67^{b}$ & $29.81 \pm 0.29^{c}$ & $26.72 \pm 1.35$ & $\begin{array}{l}34.66 \pm 1.07 \\
b\end{array}$ \\
\hline C16: 2(n-6) & $1.93 \pm 0.19^{a}$ & $1.79 \pm 0.10^{a}$ & $1.06 \pm 0.08^{b}$ & $0.37 \pm 0.01^{c}$ & - \\
\hline C16: 3(n-4) & $7.41 \pm 0.31^{d}$ & $13.45 \pm 0.50^{a}$ & $12.08 \pm 1.30^{b}$ & $6.74 \pm 0.22^{d}$ & $8.84 \pm 0.35^{c}$ \\
\hline C16: 4(n-1) & - & - & - & $1.77 \pm 0.09^{a}$ & - \\
\hline C18: 2(n-6) & - & - & $1.34 \pm 0.21^{b}$ & $1.38 \pm 0.20^{b}$ & $2.24 \pm 0.30^{\mathrm{a}}$ \\
\hline C18: 4(n-12) & - & - & - & $0.70 \pm 0.03^{a}$ & - \\
\hline C20: 4(n-6) & - & $1.28 \pm 0.11^{a}$ & $0.89 \pm 0.07^{b}$ & $0.27 \pm 0.01^{c}$ & $0.80 \pm 0.02^{b}$ \\
\hline
\end{tabular}

Different letters indicate statistically significant difference $(P<0.05$, Duncan's analysis). The data were shown as mean \pm standard deviation.

1. SFA: Saturated fatty acids.

2. MUFA: Monounsaturated fatty acids.

3. PUFA: Polyunsaturated fatty acids.

4. $n-6$ PUFAs: $n-6$ polyunsaturated fatty acids.

5. n-3 PUFAs: n-3 polyunsaturated fatty acids. 


\begin{tabular}{|c|c|c|c|c|c|}
\hline \multirow{2}{*}{$\begin{array}{l}\text { Fatty Acid } \\
\text { Profile }\end{array}$} & \multicolumn{5}{|l|}{ Proportion (\%) } \\
\hline & RL & YL & GL & BL & WL \\
\hline C20: $5(n-3)$ & $\begin{array}{l}18.21 \pm 1.70 \\
d\end{array}$ & $20.58 \pm 0.25^{c}$ & $21.27 \pm 1.89$ & $\begin{array}{l}27.77 \pm 1.27 \\
a\end{array}$ & $23.37 \pm 1.30$ \\
\hline C22: $6(n-3)$ & $1.31 \pm 0.09 a$ & $1.00 \pm 0.09 b$ & $1.02 \pm 0.08^{b}$ & $0.95 \pm 0.13 b c$ & $0.80 \pm 0.02^{c}$ \\
\hline PUFA & $28.86 \pm 1.23$ & $\begin{array}{l}38.09 \pm 0.90 \\
a b\end{array}$ & $37.66 \pm 0.93^{b}$ & $\begin{array}{l}39.95 \pm 1.66 \\
a\end{array}$ & $\begin{array}{l}36.04 \pm 0.80 \\
b\end{array}$ \\
\hline n-3 PUFA & $19.52 \pm 1.66$ & $\begin{array}{l}21.58 \pm 0.25 \\
b c\end{array}$ & $22.29 \pm 1.86^{b}$ & $\begin{array}{l}28.72 \pm 1.36 \\
a\end{array}$ & $\begin{array}{l}24.17 \pm 1.29 \\
b\end{array}$ \\
\hline n-6 PUFA & $1.93 \pm 0.19 b$ & $3.07 \pm 0.19 a$ & $3.29 \pm 0.23^{a}$ & $2.02 \pm 0.20^{b}$ & $3.04 \pm 0.29$ a \\
\hline \multicolumn{6}{|c|}{$\begin{array}{l}\text { Different letters indicate statistically significant difference }(P<0.05 \text {, Duncan's analysis). The data } \\
\text { were shown as mean } \pm \text { standard deviation. }\end{array}$} \\
\hline \multicolumn{6}{|c|}{ 1. SFA: Saturated fatty acids. } \\
\hline \multicolumn{6}{|c|}{ 2. MUFA: Monounsaturated fatty acids. } \\
\hline \multicolumn{6}{|c|}{ 3. PUFA: Polyunsaturated fatty acids. } \\
\hline \multicolumn{6}{|c|}{ 4. n-6 PUFAs: $n-6$ polyunsaturated fatty acids. } \\
\hline \multicolumn{6}{|c|}{ 5. n-3 PUFAs: $n-3$ polyunsaturated fatty acids. } \\
\hline
\end{tabular}

White light (LED) was used as the control group, and LED lights of different single colours red, yellow, blue, and green light) were used as the light source for $C$. closterium growth. All microalgal cultivation experiments were performed at a constant temperature and light incubator (PGX-330AY2-intelligent lightquality incubator, Ningbo Lifewww Technology Company, China). The cell density was adjusted to $16 \times 10^{4}$ cell ind $\cdot \mathrm{mL}^{-1}$ after inoculation to ensure the same starting points for all experimental conditions. The cultivation conditions were set as follows: static closed cultivation, shaken twice a day (8:00 and 18:00), temperature adjusted to around $20 \pm 1^{\circ} \mathrm{C}$, the light intensity controlled at $120 \pm 2 \mu \mathrm{mol} \cdot \mathrm{m}^{-2} \cdot \mathrm{s}^{-1}$, and the light and dark cycle maintained at (L: D) 12:12 [13,15]. The light intensity was measured by an illuminometer (GM1030C, BENETECH, Shenzhen Jumaoyuan Science and Technology Company, China).

\section{Growth determination}

Three biological replicates were set-up for each different light quality condition. The cells were harvested for further processing at day 7 after inoculation, and the cell density was measured by Andreasson method [45]. The growth rate (GR) was estimated by linear regression using the following expression: GR $=\left(\ln N_{t}-\ln N_{0}\right) / t(\mu / d)$ 
where $\mathrm{N}_{\mathrm{t}}$ is the number of cells measured each day (from the first to sixth day of culturing) and $\mathrm{N}_{0}$ is the initial cell density.

\section{Chlorophyll a determination}

The chlorophylls were measured as described by the acetone method [46]. Ten millilitres of algal liquid were harvested by centrifugation into a $50 \mathrm{~mL}$ centrifuge tube and washed with distilled water to remove extra salt. The samples were leached with $10 \mathrm{~mL} 90 \%$ acetone (hatched at $4^{\circ} \mathrm{C}, \mathrm{v} / \mathrm{v}$ ), stored in a refrigerator for $24 \mathrm{~h}$. After centrifugation at $12000 \mathrm{rpm}$ for $20 \mathrm{~min}$ at $4^{\circ} \mathrm{C}$, pigment supernatant was obtained for the determination of absorbance at wavelengths of $640 \mathrm{~nm}$ and $630 \mathrm{~nm}$, and $90 \%$ acetone was used as the blank control. The chlorophylls were calculated according to the following formula:

Chlorophyll a (ug/ind) $=(11.47$ A664-0.40A630) $/ C$

Chlorophyll c (ug/ind) $=(24.36$ A630-3.73A664) $/ C$

( $C$ is the density of algal cells at exponential phase)

\section{Total lipid and fatty acid profile determination}

The algal cells were harvested by centrifugation into a $50 \mathrm{~mL}$ centrifuge tube and washed with distilled water to remove extra salt. The samples were stored in a refrigerator, frozen at $-20^{\circ} \mathrm{C}$ for one day and lyophilized (FD5-3T Vertical Freeze Dryer, Gold-SIM) for 2 days. Then, $1000 \mathrm{mg}$ of algal powder was weighed, and total lipids was extracted by the Bligh and Dyer method [47] and rotary evaporation to a constant weight to measure lipid content.

The fatty acid profile of algae was measured as described by [48]. Fatty acids were determined from the lipid samples. The solutions containing 5-6\% (w/v) $\mathrm{KOH}$ dissolved in $\mathrm{CH}_{3} \mathrm{OH}$ and $14 \% \mathrm{BF}_{3}-\mathrm{CH}_{3} \mathrm{OH}$ solution were added to the crude lipid samples for saponification and methylation and rotary evaporation. The samples were extracted to obtain supernatant with n-hexane by centrifugation. The fatty acid profile was separated and identified using GC-MS (Agilent 7890B single bond 7000C, GC-MS, Agilent Technologies, Santa Clara, CA, USA) equipped with a Vocol fused-silica capillary column (60 $\mathrm{m} \times$ $0.32 \mathrm{~mm}$ i.d., $0.25 \mu \mathrm{m}$ film thickness; Supelco, Bellefonte, PA, USA). We confirmed the chemical structure formulae of fatty acids through Chem Spider (http://www.chemspider.com; Royal Society of Chemistry, London, UK). Relative quantities were expressed as percentage weight of total fatty acids in each sample (\% total fatty acid).

\section{Statistical analysis}

The results were analysed based on the data from at least three different algal cultivations. Experimental results were expressed as the mean value \pm SD. The differences between groups were tested for

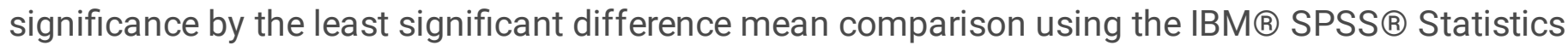
software program (version 23). The relationship between variables was determined by one-way ANOVA at 
a significance level of 0.05 using a Duncan's test. The graphs were drawn in Origin 2018. Correlation analysis and principal component analysis (PCA) were visualized using the R Studio (4.0.3).

\section{Declarations}

\section{Ethics approval and consent to participate}

This article does not contain any studies with human participants or animals performed by any of the authors.

\section{Consent for publication}

Not applicable

\section{Competing interests}

The authors declare that they have no competing interests.

\section{Funding}

This research was funding by the Major Projects in Zhejiang Province (2019C02055) and partly sponsored Public Welfare Fund in Ningbo (202002N3044).

\section{Authors' contributions}

$\mathrm{CZ}$ and $\mathrm{YW}$ prepared the microalgal strain and performed the algal cultivation. $\mathrm{ZZ}$ and $\mathrm{ZW}$ tested the growth rate and chlorophylls contents. CFC and YW performed total lipids tests and fatty acids analyses. $\mathrm{RP}$ and XJ supervised the work. ZX provided the instruments and incubators for these experiments. The manuscript was written by $\mathrm{CZ}$ with contributions from RP. All authors made comments on the manuscript and approved the final version. Figures were prepared by CFC and RP. All authors read and approved the final manuscript.

\section{Author details}

Key Laboratory of Applied Marine Biotechnology, School of Marine Sciences, Ningbo University, Ningbo, Zhejiang Province 315211, PR China

\section{Acknowledgements}

The authors would like to thank the GC-MS for the fatty acid analysis, provided by large-scale equipment platform (School of Marine Sciences, Ningbo University), as well as thank Ruiping Xue, Weixia Zhang and Peng Zhou (Ningbo University) for their assistance with caring for microalgae cultivation and conserved.

\section{Availability of data and materials}


The datasets used and/or analyzed during the current study are available from the corresponding author on reasonable request.

\section{References}

1. Avinash A, Sasikumar P, Pugazhendhi A. Analysis of the limiting factors for large scale microalgal cultivation: A promising future for renewable and sustainable biofuel industry. Renew Sustain Energy Rev. 2020;134:110250. https://doi.org/10.1016/j.rser.2020.110250.

2. Yesilyurt MK, Cesur C, Aslan V, Yilbasi Z. The production of biodiesel from safflower (Carthamus tinctorius $\mathrm{L}$.) oil as a potential feedstock and its usage in compression ignition engine: $\mathrm{A}$ comprehensive review. Renew Sustain Energy Rev. 2020;119:109574. http://dx.doi.org/10.1016/j.rser.2019.109574.

3. Yin Z, Zhu L, Li S, Hu T, Chu R, Mo F, et al. A comprehensive review on cultivation and harvesting of microalgae for biodiesel production: Environmental pollution control and future directions. Bioresour Technol. 2020;301:122804. https://doi.org/10.1016/j.biortech.2020.122804.

4. Kazemi Shariat Panahi H, Tabatabaei M, Aghbashlo M, Dehhaghi M, Rehan M, Nizami A-S. Recent updates on the production and upgrading of bio-crude oil from microalgae. Bioresour Technol Reports. 2019;7:100216. https://doi.org/10.1016/j.biteb.2019.100216.

5. Yan C, Zhang L, Luo X, Zheng Z. Effects of various LED light wavelengths and intensities on the performance of purifying synthetic domestic sewage by microalgae at different influent $\mathrm{C} / \mathrm{N}$ ratios. Ecol Eng. 2013;51:24-32. http://dx.doi.org/10.1016/j.ecoleng.2012.12.051.

6. Choi S-A, Oh Y-K, Jeong M-J, Kim SW, Lee J-S, Park J-Y. Effects of ionic liquid mixtures on lipid extraction from Chlorella vulgaris. Renew Energy. 2014;65:169-74. http://dx.doi.org/10.1016/j.renene.2013.08.015.

7. Ma Y, Wang Z, Yu C, Yin Y, Zhou G. Evaluation of the potential of 9 Nannochloropsis strains for biodiesel production. Bioresour Technol. 2014;167:503-9. http://dx.doi.org/10.1016/j.biortech.2014.06.047.

8. Yang Y, Weathers P. Red light and carbon dioxide differentially affect growth, lipid production, and quality in the microalga, Ettlia oleoabundans. Appl Microbiol Biotechnol. 2014;99:489-99. http://dx.doi.org/10.1007/s00253-014-6137-1.

9. Patelou M, Infante C, Dardelle F, Randewig D, Kouri ED, Udvardi MK, et al. Transcriptomic and metabolomic adaptation of Nannochloropsis gaditana grown under different light regimes. Algal Res. 2020;45:101735. https://doi.org/10.1016/j.algal.2019.101735.

10. Li D, Yuan Y, Cheng D, Zhao Q. Effect of light quality on growth rate, carbohydrate accumulation, fatty acid profile and lutein biosynthesis of Chlorella sp. AE10. Bioresour Technol. 2019;291:121783. https://doi.org/10.1016/j.biortech.2019.121783.

11. Sorokin C, Krauss RW. The Effects of Light Intensity on the Growth Rates of Green Algae. Plant Physiol. 1958;33(2):109-13. http://dx.doi.org/10.1104/pp.33.2.109. 
12. Chiang C, Bånkestad D, Hoch G. Reaching Natural Growth: The Significance of Light and Temperature Fluctuations in Plant Performance in Indoor Growth Facilities. Plants. 2020;9(10):1312. http://dx.doi.org/10.3390/plants9101312.

13. Shi J, Pan K, Wang X, Chen F, Zhou M, Zhu B, et al. Hierarchical recognition on the taxonomy of Nitzschia closterium f. minutissima. Chinese Sci Bull. 2008;53(2):245-50. http://dx.doi.org/10.1007/s11434-008-0080-4.

14. De Miranda M, Gaviano M, Serra E. Changes in the cell size of the diatom Cylindrotheca closterium in a hyperhaline pond. Chem Ecol. 2005;21(1):77-81. http://dx.doi.org/10.1080/02757540512331323962.

15. Suman K, Kiran T, Devi UK, Sarma NS. Culture medium optimization and lipid profiling of Cylindrotheca, a lipid- and polyunsaturated fatty acid-rich pennate diatom and potential source of eicosapentaenoic acid. Bot Mar. 2012;55(3):289-99. http://dx.doi.org/10.1515/bot-2011-0076.

16. Wang S, Sirbu D, Thomsen L, Kuhnert N, Ullrich MS, Thomsen C. Comparative lipidomic studies of Scenedesmus sp. (Chlorophyceae) and Cylindrotheca closterium (Bacillariophyceae) reveal their differences in lipid production under nitrogen starvation. J Phycol. 2019;55(6):1246-57. http://dx.doi.org/10.1111/jpy.12887.

17. Brennan B, Regan F. In-situ lipid and fatty acid extraction methods to recover viable products from Nannochloropsis sp. Sci Total Environ. 2020;748:142464. https://doi.org/10.1016/j.scitotenv.2020.142464.

18. Li Y, Li R, Yi X. Effects of light quality on growth rates and pigments of Chaetoceros gracilis (Bacillariophyceae). J Oceanol Limnol. 2020;38(3):795-801. http://dx.doi.org/10.1007/s00343-0199171-0.

19. García-López DA, Olguín EJ, González-Portela RE, Sánchez-Galván G, De Philippis R, Lovitt RW, et al. A novel two-phase bioprocess for the production of Arthrospira (Spirulina) maxima LJGR1 at pilot plant scale during different seasons and for phycocyanin induction under controlled conditions. Bioresour Technol. 2020;298:122548. https://doi.org/10.1016/j.biortech.2019.122548.

20. Wang C-Y, Fu C-C, Liu Y-C. Effects of using light-emitting diodes on the cultivation of Spirulina platensis. Biochem Eng J. 2007;37(1):21-5. http://dx.doi.org/10.1016/j.bej.2007.03.004.

21. Katz JJ, Norris JR, Shipman LL, Thurnauer MC, Wasielewski MR. Chlorophyll Function in the Photosynthetic Reaction Center. Annu Rev Biophys Bioeng. 1978;7(1):393-434. http://dx.doi.org/10.1146/annurev.bb.07.060178.002141.

22. Fortunato $A E$, Jaubert $M$, Enomoto $G$, Bouly JP, Raniello R, Thaler $M$, et al. Diatom phytochromes reveal the existence of far-red-light-based sensing in the ocean. Plant Cell. 2016;28(3):616-28. http://dx.doi.org/10.1105/tpc.15.00928.

23. Izzo LG, Hay Mele B, Vitale L, Vitale E, Arena C. The role of monochromatic red and blue light in tomato early photomorphogenesis and photosynthetic traits. Environ Exp Bot. 2020;179:104195. https://doi.org/10.1016/j.envexpbot.2020.104195. 
24. Kosugi M, Ozawa S-I, Takahashi Y, Kamei Y, Itoh S, Kudoh S, et al. Red-shifted chlorophyll a bands allow uphill energy transfer to photosystem II reaction centers in an aerial green alga, Prasiola crispa, harvested in Antarctica. Biochim Biophys Acta - Bioenerg. 2020;1861(2):148139. https://doi.org/10.1016/j.bbabio.2019.148139.

25. Mercado JM, Sánchez-Saavedra MDP, Correa-Reyes G, Lubián L, Montero O, Figueroa FL. Blue light effect on growth, light absorption characteristics and photosynthesis of five benthic diatom strains. Aquat Bot. 2004;78(3):265-77. http://dx.doi.org/10.1016/j.aquabot.2003.11.004.

26. Enomoto G, Ikeuchi M. Blue-/Green-Light-Responsive Cyanobacteriochromes Are Cell Shade Sensors in Red-Light Replete Niches. iScience. 2020;23(3):100936. https://doi.org/10.1016/j.isci.2020.100936.

27. Ruivo M, Amorim A, Cartaxana P. Effects of growth phase and irradiance on phytoplankton pigment ratios: implications for chemotaxonomy in coastal waters. J Plankton Res. 2011;33(7):1012-22. https://academic.oup.com/plankt/article-lookup/doi/10.1093/plankt/fbr019.

28. Rijstenbil J. Effects of UVB radiation and salt stress on growth, pigments and antioxidative defence of the marine diatom Cylindrotheca closterium. Mar Ecol Prog Ser. 2003;254:37-48. http://www.intres.com/abstracts/meps/v254/p37-48/.

29. Garrido L, Brunet C, Rodr F. Pigment variations in Emiliania huxleyi (CCMP370) as a response to changes in light intensity or quality. 2016;18(12):4412-4425.

30. KANA TM, GEIDER RJ. CRITCHLEY C. Regulation of photosynthetic pigments in micro-algae by multiple environmental factors: a dynamic balance hypothesis. New Phytol. 1997;137(4):629-38. http://doi.org/10.1046/j.1469-8137.1997.00857.x.

31. Li X, Slavens S, Crunkleton DW, Johannes TW. Interactive effect of light quality and temperature on Chlamydomonas reinhardtii growth kinetics and lipid synthesis. Algal Res. 2020;53:102127. https://doi.org/10.1016/j.algal.2020.102127.

32. Page RA, Okada S, Harwood JL. Acetyl-CoA carboxylase exerts strong flux control over lipid synthesis in plants. Biochim Biophys Acta (BBA)/Lipids Lipid Metab. 1994;1210(3):369-72. http://doi.org/10.1016/0005-2760(94)90242-9.

33. Hultberg M, Jönsson HL, Bergstrand KJ, Carlsson AS. Impact of light quality on biomass production and fatty acid content in the microalga Chlorella vulgaris. Bioresour Technol. 2014;159:465-7. http://dx.doi.org/10.1016/j.biortech.2014.03.092.

34. Ren J, Guo S, Xu C, Yang C, Ai W, Tang Y, et al. Effects of different carbon dioxide and LED lighting levels on the anti-oxidative capabilities of Gynura bicolor DC. Adv Sp Res. 2014;53(2):353-61. http://dx.doi.org/10.1016/j.asr.2013.11.019.

35. Mittler R. Oxidative stress, antioxidants and stress tolerance. Trends Plant Sci. 2002;7(9):405-10. http://doi.org/10.1016/S1360-1385(02)02312-9.

36. Radakovits R, Eduafo PM, Posewitz MC. Genetic engineering of fatty acid chain length in Phaeodacty/um tricornutum. Metab Eng. 2011;13(1):89-95. http://dx.doi.org/10.1016/j.ymben.2010.10.003. 
37. Afitlhile M, Duffield-Duncan K, Fry M, Workman S, Hum-Musser S, Hildebrand D. The toc 132 toc 120 heterozygote mutant of Arabidopsis thaliana accumulates reduced levels of hexadecatrienoic acid. Plant Physiol Biochem. 2015;96:426-35. http://doi.org/10.1016/j.plaphy.2015.09.006.

38. Wang $X$, Zhang P, Wu Y, Zhang L. Effect of light quality on growth, ultrastructure, pigments, and membrane lipids of Pyropia haitanensis. J Appl Phycol. 2020;32(6):4189-97. http://doi.org/10.1007/s10811-020-02264-4.

39. Leyland B, Boussiba S, Khozin-Goldberg I. A Review of Diatom Lipid Droplets. Biology (Basel). 2020;9(2):12-23. http://doi.org/10.3390/biology9020038.

40. Erwin J, Hulanicka D, Bloch K. Comparative aspects of unsaturated fatty acid synthesis. Comp Biochem Physiol. 1964;12(2):191-207. http://doi.org/10.1016/0010-406X(64)90174-4.

41. Xia Y, Zhang YT, Sun JY, Huang H, Zhao Q, Ren LJ. Strategies for enhancing eicosapentaenoic acid production: From fermentation to metabolic engineering. Algal Res. 2020;51:102038. https://doi.org/10.1016/j.algal.2020.102038.

42. Talarico L, Maranzana G. Light and adaptive responses in red macroalgae: An overview. J Photochem Photobiol B Biol. 2000;56(1):1-11. http://doi.org/10.1016/S1011-1344(00)00046-4.

43. Ueno Y, Aikawa S, Kondo A, Akimoto S. Adaptation of light-harvesting functions of unicellular green algae to different light qualities. Photosynth Res. 2019;139:145-54. https://doi.org/10.1007/s11120-018-0523-y.

44. Chen Q, Jiang X, Han Q, Sheng P, Chai Y, Peng R, et al. Growth, calcium content, proximate composition, and fatty acid composition of triangle sail mussel (Hyriopsis cumingii) fed five different microalgal diets. Aquaculture. 2021;530:735719. https://doi.org/10.1016/j.aquaculture.2020.735719.

45. Andreasson KIM, Wängberg S-Å. Reduction in growth rate in Phaeodactylum tricornutum (Bacillariophyceae) and Dunaliella tertiolecta (Chlorophyceae) induced by UV-B radiation. J Photochem Photobiol B Biol. 2007;86(3):227-33. http://doi.org/10.1016/j.jphotobiol.2006.10.005.

46. Jeffrey SW, Humphrey GF. New spectrophotometric equations for determining chlorophylls $a, b, c 1$ and $\mathrm{c} 2$ in higher plants, algae and natural phytoplankton. Biochem Physiol der Pflanz. 1975;167(2):191-4. http://dx.doi.org/10.1016/S0015-3796(17)30778-3.

47. Bligh EG, Dyer WJ. A rapid method of total lipid extraction and purification. Can J Biochem Physiol. 1959;37(8):911-7. http://doi.org/10.1139/059-099.

48. Reiser S, Somerville C. Isolation of mutants of Acinetobacter calcoaceticus deficient in wax ester synthesis and complementation of one mutation with a gene encoding a fatty acyl coenzyme $A$ reductase. J Bacteriol. 1997;179(9):2969-75. http://doi.org/10.1128/jb.179.9.2969-2975.1997.

\section{Figures}




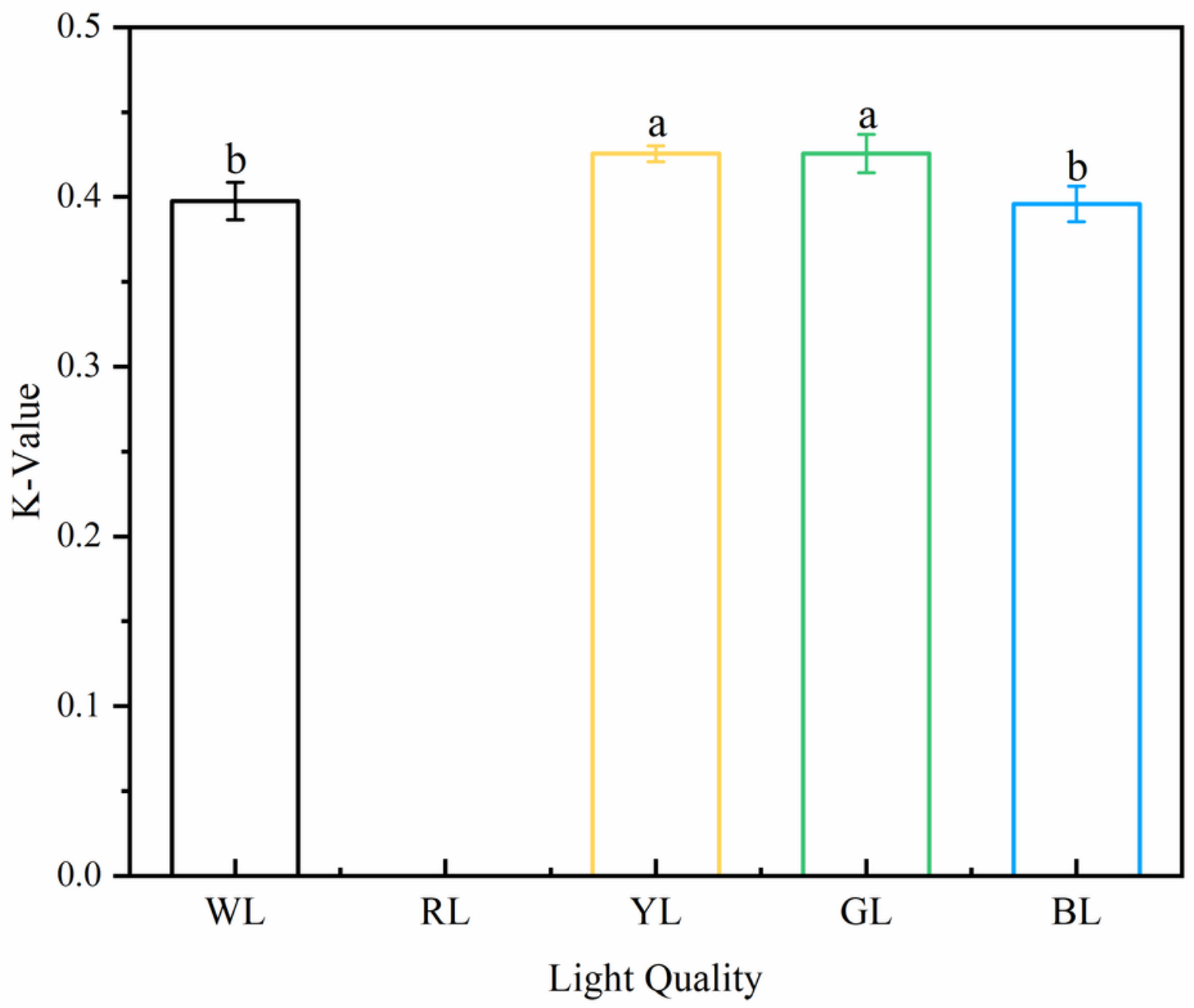

Figure 1

Growth of C. closterium cultures grown under different light source as well as control conditions. RL (red light), BL (blue light), GL (green light), YL (yellow light) and WL (white light as control group). Different letters indicate statistically significant difference $(P<0.05$, Duncan's analysis). Data are shown as mean $+/$-SD of 3 biological repeats $(n=3)$. 


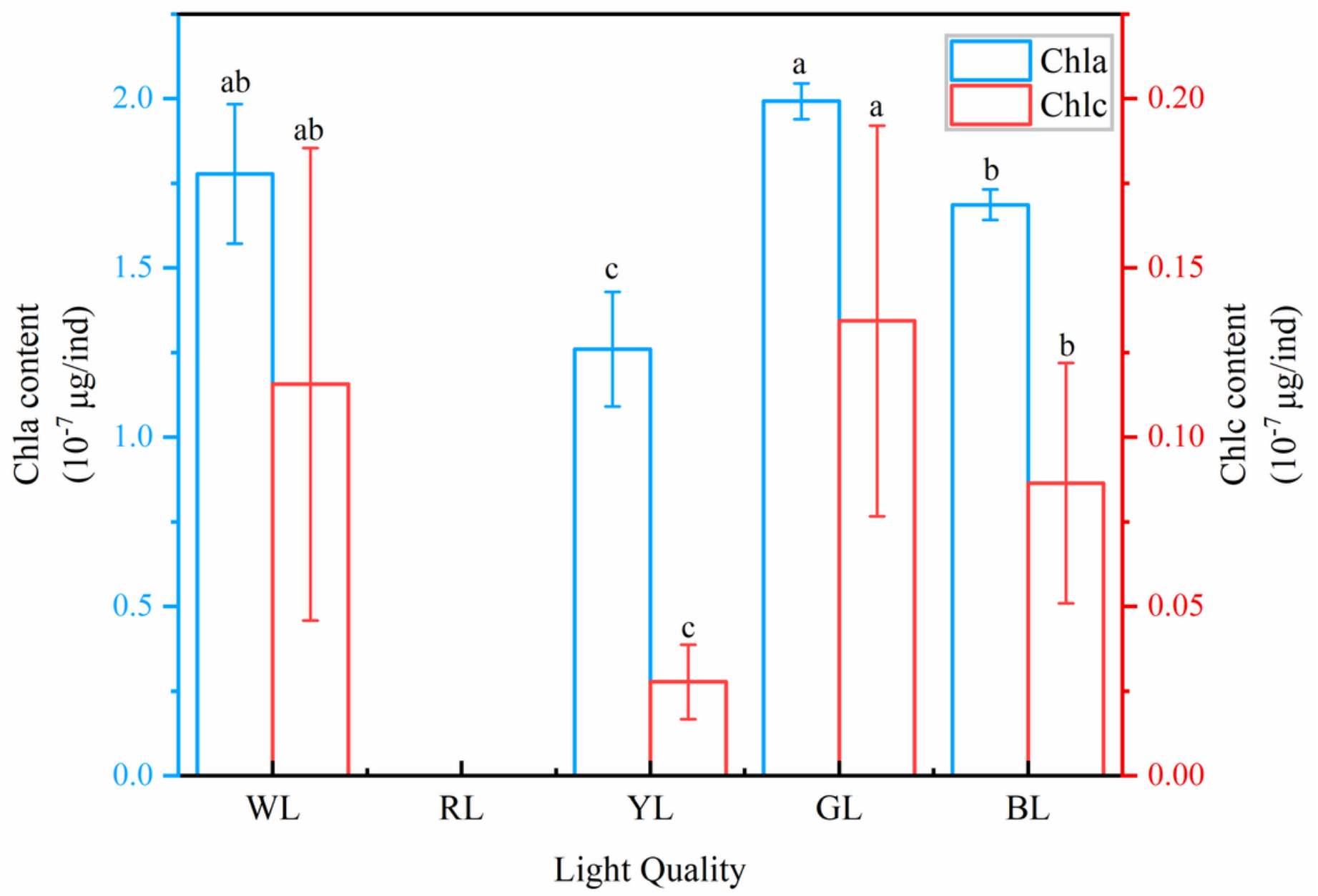

Figure 2

Chls concentration of $\mathrm{C}$. closterium cultures grown under different light source as well as control conditions. RL (red light), BL (blue light), GL (green light), YL (yellow light) and WL (white light as control group). Different letters indicate statistically significant difference $(P<0.05$, Duncan's analysis). 


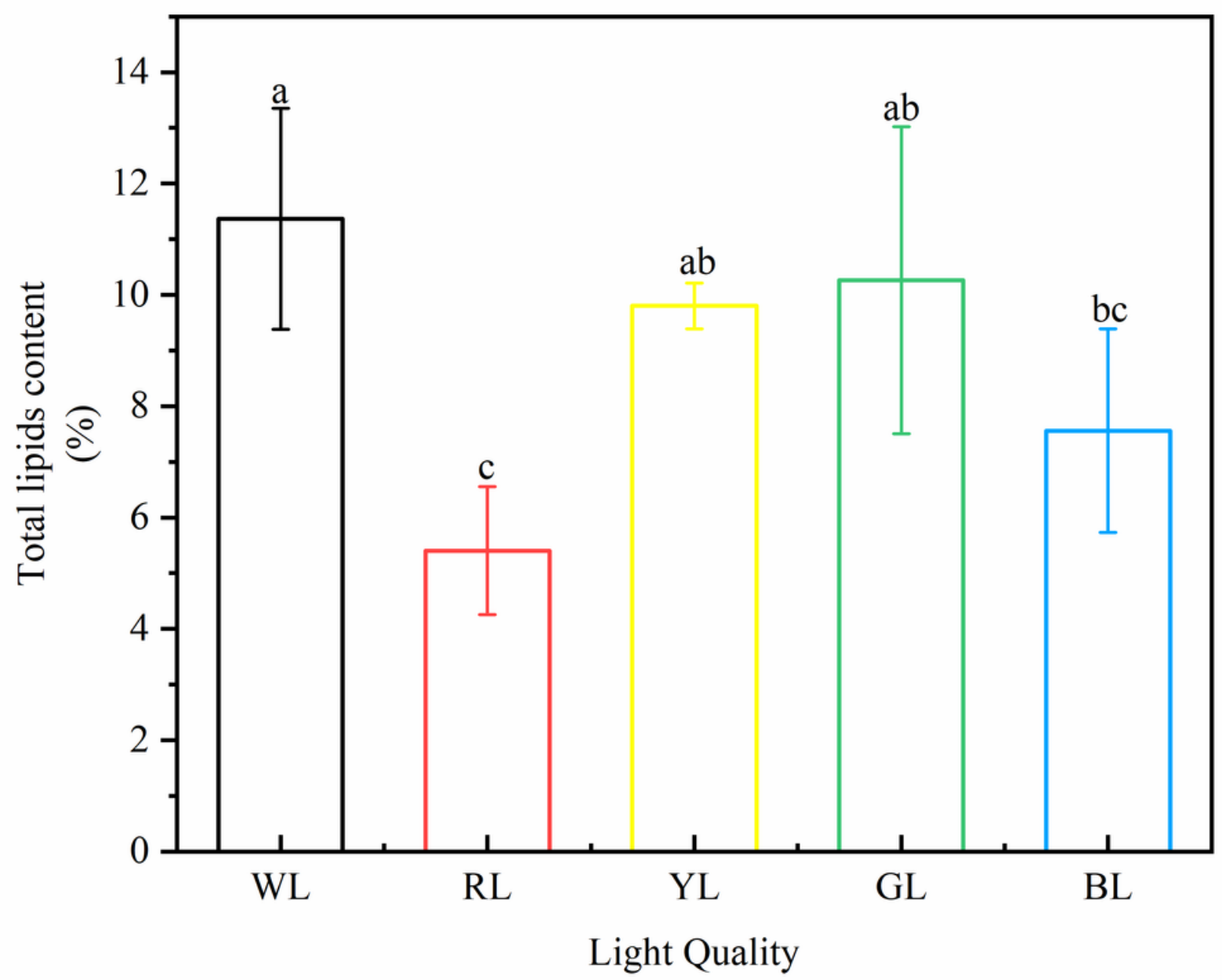

Figure 3

Lipid Content of C. closterium cultures grown under different light source as well as control conditions. RL (red light), BL (blue light), GL (green light), YL (yellow light) and WL (white light as control group). Different letters indicate statistically significant difference $(P<0.05$, Duncan's analysis). 

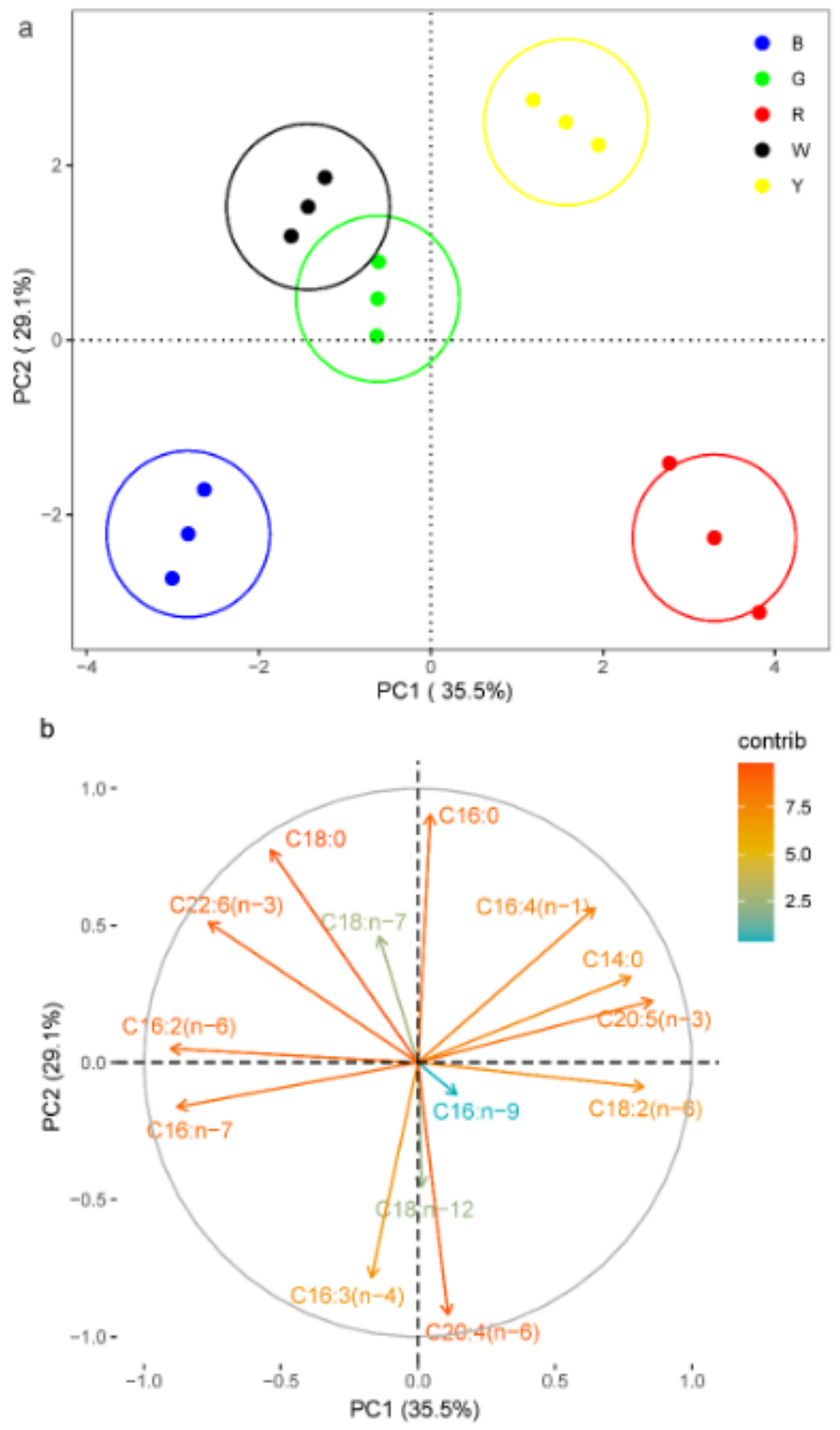

Figure 4

Principal component analysis (PCA) based on fatty acid compositions of $\mathrm{C}$. closterium cultures under five light sources. Fatty acid compositions from five light-source groups, R (red light), B (blue light), G (green light), Y (yellow light) and $W$ (white light as control group). The upper panel (a) shows factor coefficients plot (a), while the lower panel (b) shows the loading plot for the corresponding fatty acids' contribution to the scores plot. 


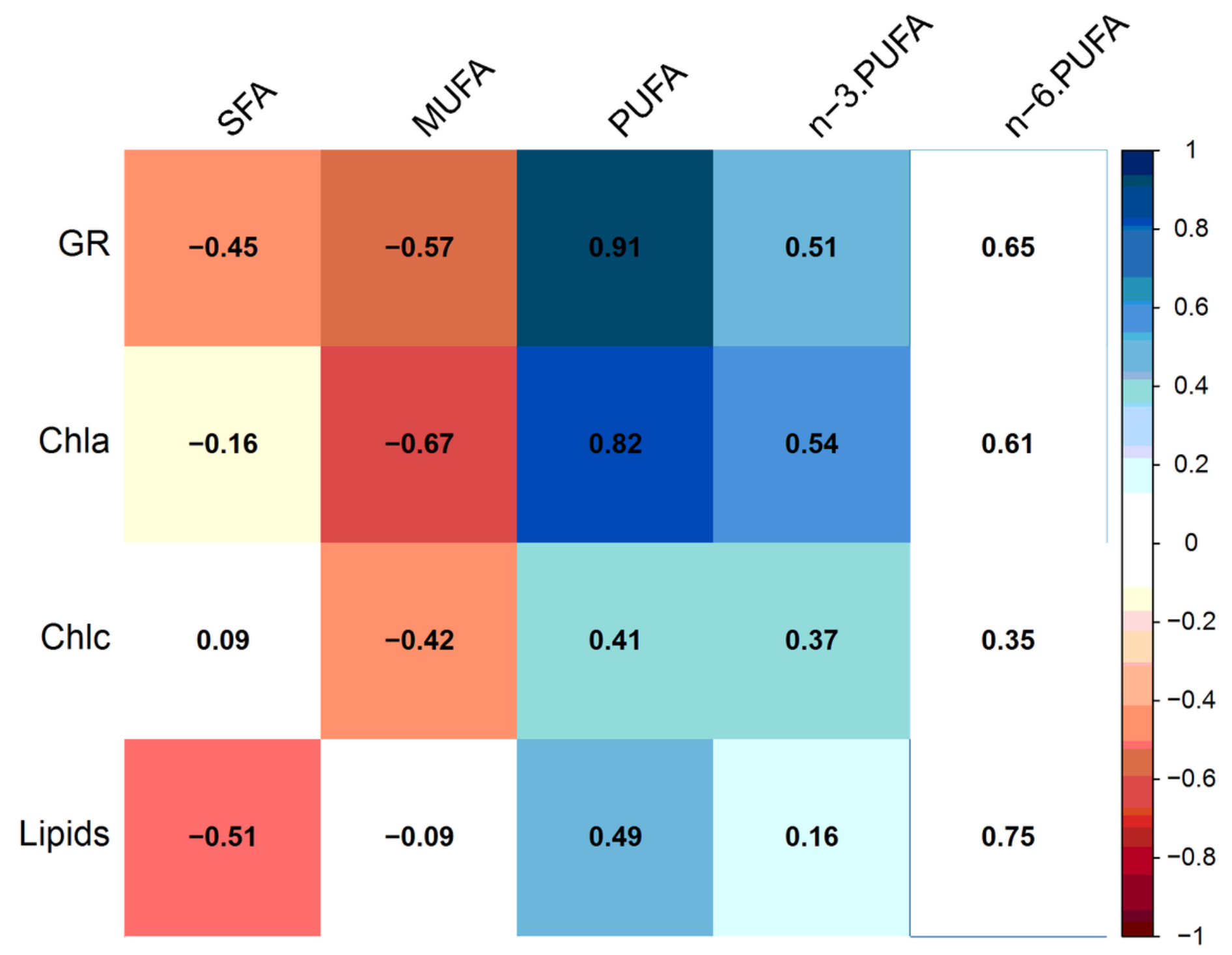

\section{Figure 5}

Correlation analysis of GR (growth rate), Chla (chlorophyll a), Chlc (chlorophyll c), Lipids (total lipids content), SFA (saturated fatty acid), MUFA (monounsaturated fatty acid), PUFA (polyunsaturated fatty acid), n-3 PUFA, and n-6 PUFA among the $\mathrm{C}$. closterium cultures grown under different light source. The color shades represent the numerical size of the correlation coefficient. $|r| \geq 0.8$, very strong correlation; $\mathbb{Z}$

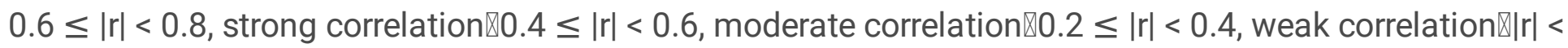
0.2 , no correlation. 